\title{
Long non-coding RNA Xist regulates oocyte loss via suppressing miR-23b-3p/miR-29a-3p maturation and upregulating STX17 in perinatal mouse ovaries
}

\author{
Meng Zhou ${ }^{1}$, Xiaoqiu Liu' ${ }^{2}$ E. Qiukai ${ }^{1}$, Yanxing Shang ${ }^{1}$, Xiaoqian Zhang ${ }^{1}$, Shuting Liu ${ }^{1}$ and Xuesen Zhang ${ }^{1}$
}

\begin{abstract}
The fecundity of female mammals is resolved by the limited size of the primordial follicle (PF) pool formed perinatally. The establishment of PF pool is accompanied by a significant programmed oocyte death. Long non-coding RNAs (IncRNA) are central modulators in regulating cell apoptosis or autophagy in multiple diseases, however, the significance of IncRNAs governing perinatal oocyte loss remains unknown. Here we find that Yin-Yang 1 (YY1) directly binds to the IncRNA X-inactive-specific transcript (Xist) promoter and facilitates Xist expression in the perinatal mouse ovaries. Xist is highly expressed in fetal ovaries and sharply downregulated along with the establishment of PF pool after birth. Gain or loss of function analysis reveals that Xist accelerates oocyte autophagy, mainly through binding to pre-miR-23b or pre-miR-29a in the nucleus and preventing the export of pre-miR-23b/pre-miR-29a to the cytoplasm, thus resulting in decreased mature of miR-23b-3p/miR-29a-3p expression and upregulation miR-23b-3p/miR-29a-3p cotarget, STX17, which is essential for timely control of the degree of oocyte death in prenatal mouse ovaries. Overall, these findings identify Xist as a key non-protein factor that can control the biogenesis of miR-23b-3p/miR-29a-3p, and this YY1-Xist-miR-23b-3p/miR-29a-3p-STX17 regulatory axis is responsible for perinatal oocyte loss through autophagy.
\end{abstract}

\section{Introduction}

The establishment of the primordial follicle (PF) pool represents the first stage of folliculogenesis and this nonrenewable PF resource is crucial for maintaining female reproductive life ${ }^{1}$. As the principal functional reproductive unit that provides mature oocytes, the population of PFs is perinatally established, in a process termed cyst breakdown ${ }^{2}$. Ensuring proper primordial follicle generation and early follicle development is under precise control $^{1}$. However, multiple factors can induce excessive loss or overactivation of PFs in the PF pool, which is closely related to primary premature ovarian insufficiency (POI) and even to female infertility ${ }^{3,4}$. A better understanding of

\footnotetext{
Correspondence: Xuesen Zhang (zhang_xuesen@163.com)

${ }^{1}$ State Key Laboratory of Reproductive Medicine, Nanjing Medical University, 211166 Nanjing, China

${ }^{2}$ Department of Microbiology, Key Laboratory of Pathogen Biology of Jiangsu Province, Nanjing Medical University, 211166 Nanjing, China

These authors contributed equally: Meng Zhou, Xiaoqiu Liu

Edited by V. Dötsch
}

the regulatory mechanisms underlying PF pool formation will provide us with deep insights into the potential pathogenic mechanism of $\mathrm{POI}^{5-7}$.

Till now, the main studies on PF pool formation or even the pathogenesis of POI associated with PF formation are restricted to protein-coding genes ${ }^{8,9}$. However, only a small proportion of POI cases can be interpreted by causative genetic defects, such as chromosomal abnormalities and gene mutations ${ }^{4,8,10,11}$. Eukaryotic genomes have a wide range of transcriptional properties, producing a large number of RNA transcripts. Among them, only $<2 \%$ of these RNAs will serve as mRNA templates, and the remaining part of transcripts are non-coding RNAs (ncRNAs). Obviously, there is a lack of study on the roles of ncRNAs, especially long non-coding RNAs (lncRNAs) at the early stage of folliculogenesis, since these ncRNAs have recently been proved to play essential roles in both physiological and pathological conditions in female reproduction ${ }^{9}$.

\section{(c) The Author(s) 2021}

(c) (i) Open Access This article is licensed under a Creative Commons Attribution 4.0 International License, which permits use, sharing, adaptation, distribution and reproduction cc) in any medium or format, as long as you give appropriate credit to the original author(s) and the source, provide a link to the Creative Commons license, and indicate if changes were made. The images or other third party material in this article are included in the article's Creative Commons license, unless indicated otherwise in a credit line to the material. If material is not included in the article's Creative Commons license and your intended use is not permitted by statutory regulation or exceeds the permitted use, you will need to obtain permission directly from the copyright holder. To view a copy of this license, visit http://creativecommons.org/licenses/by/4.0/. 
LncRNAs are a category of ncRNAs with a length of over 200 nucleotides without protein-coding poten$\mathrm{tial}^{12}$. They can function as a signal, guide, decoy, microRNA sponging, or scaffold to modulate gene expression at transcription and post-transcription levels ${ }^{13,14}$. Several recent studies have linked lncRNAs to the process of folliculogenesis ${ }^{15-18}$, and dysregulation of their expression can cause ovarian diseases, including $\mathrm{POI}^{16,17,19,20}$. Besides, transcriptional profiling of lncRNAs in ovarian cortical tissues from women with POI has recently been performed, and 20 differently expressed lncRNAs were identified to be involved in ovarian follicular development ${ }^{21}$. However, whether IncRNAs are participating in the PF pool formation is nearly completely unknown. A recent transcriptome analysis of lncRNA expression in human primordial, primary and small antral follicles was performed and lncRNA X-inactive-specific transcript (Xist) is revealed to be highly transcribed throughout the stages tested, suggesting that Xist has emerged from the dormant primordial stage of human follicle development ${ }^{15}$. But its biological function and the molecular mechanism underlying the formation of the PF pool have not been clarified.

MicroRNAs (miRNAs) are another class of short ncRNAs with a length of 20-24 nucleotides, which are generated through the initial nuclear cleavage and subsequent cytoplasmic cleavage events ${ }^{22,23}$. The miRNA gene is initially transcribed from the genome into primary transcript (pri-miRNA), followed by cleavage into $\sim 70$ nucleotide precursors miRNA (premiRNAs). The pre-miRNA is then exported into the cytoplasm where it is further cleaved into an RNA duplex analogous. The mature miRNA is finally generated once one strand is discarded. It is well acknowledged that miRNAs can negatively control the expression of target genes in a wide range of cell signaling pathways related to different physiological and pathological processes ${ }^{24,25}$, including PF formation and even $\mathrm{POI}^{26-29}$. However, these studies focus more on how these miRNAs regulate their downstream targets and participate in the regulation of PF formation, and reports of miRNA biogenesis and regulation are missing.

In this study, we showed a time-specific decrease in Xist levels from the fetal to neonatal period in the ovaries, which correlates with the upregulated expression of $m i R$ $23 b-3 p / m i R-29 a-3 p$, and a stepwise decrease of STX17. We then demonstrated that Xist can block miR-23b-3p/ $m i R-29 a-3 p$ processing, resulting in decreased mature of $m i R-23 b-3 p / m i R-29 a-3 p$ expression, leading to upregulation of STX17, which is essential for timely control of the degree of oocyte death induced by autophagy in prenatal mouse ovaries.

\section{Results}

\section{Xist is essential for PF pool formation in the perinatal} mouse ovaries

To address the physiological significance of Xist during the establishment of PF pool in the ovaries of newborn mice, we first showed by qRT-PCR analyses that Xist transcripts started to decrease in a time-specific manner from $18.5 \mathrm{~d}$ postcoitus (dpc) to $4.5 \mathrm{~d}$ postpartum (dpp) (Fig. 1A). This dynamic change of Xist just coincides with the timing of massive oocyte loss, indicating that Xist may be involved in regulating oocyte loss. To assess this possibility, gain or loss of function experiments were carried out into $0.5 \mathrm{dpp}$ ovaries by separately transfecting with Xist siRNA (siXist) and the control siRNA (siNC), or XistpcDNA3.1 (Xist OE) and the empty control (NC). The qRT-PCR analysis confirmed that siXist transfection decreased, and Xist overexpression increased Xist expression in neonatal mouse ovaries after $96 \mathrm{~h}$ of in vitro culture (Fig. 1B, C). Subsequent histological analysis showed a significant increase of oocyte number upon Xist knockdown compared with the control (Fig. 1D), while Xist OE resulted in significantly less available oocytes than did NC (Fig. 1E). In agreement with the changes of oocyte quantification, expression of the genes essential for PF formation also showed an increase in siXist-treated ovaries (Fig. 1F). Alternatively, expression of these genes was inhibited upon Xist OE (Fig. 1G). These results indicate that Xist may be required for oocyte loss in the newborn ovaries and a decrease of Xist is indispensable for PF pool formation.

\section{Xist promotes perinatal oocyte loss mainly through oocyte autophagy}

It is well acknowledged that apoptosis and autophagy are involved in the process of oocyte death during PF pool establishment $^{30,31}$. To clarify whether Xist is involved in the programmed oocyte death through apoptosis and autophagy, we first performed TUNEL analysis to evaluate oocyte apoptosis. Overexpression of Xist did not increase the number of TUNEL-positive cells, suggesting that oocyte apoptosis may not be the major cause of oocyte loss upon Xist OE (Fig. 2A). As autophagy is also reported to be active during follicular assembly and contributes to perinatal oocyte loss ${ }^{5}$, we, therefore, tested whether Xist could induce autophagy in perinatal mouse ovaries. As the microtubule-associated protein light chain 3B (LC3B) is a marker of autophagosomes, we observed more LC3B puncta presented in the cytoplasm of Xist-overexpressed oocytes (Fig. 2B). We then incubated the $0.5 \mathrm{dpp}$ ovaries with 3MA, an autophagy inhibitor, and 3MA treatment nearly completely inhibited LC3B protein expression, while Xist overexpression could slightly eliminate the negative effect of 3MA on LC3B expression (Fig. 2C). Furthermore, TEM analysis identified the large double- 


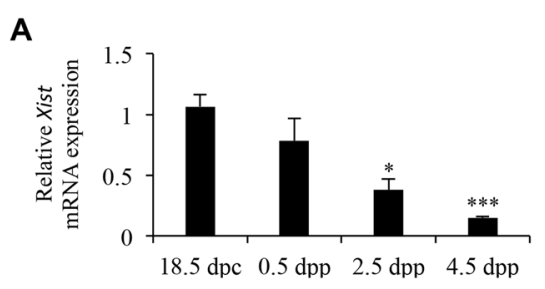

B

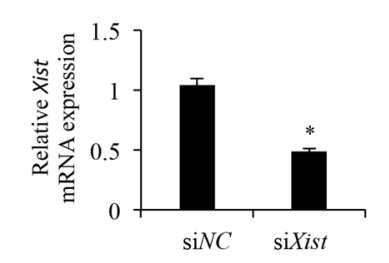

C

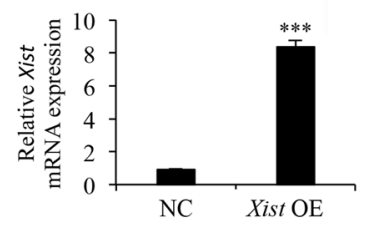

\section{F}
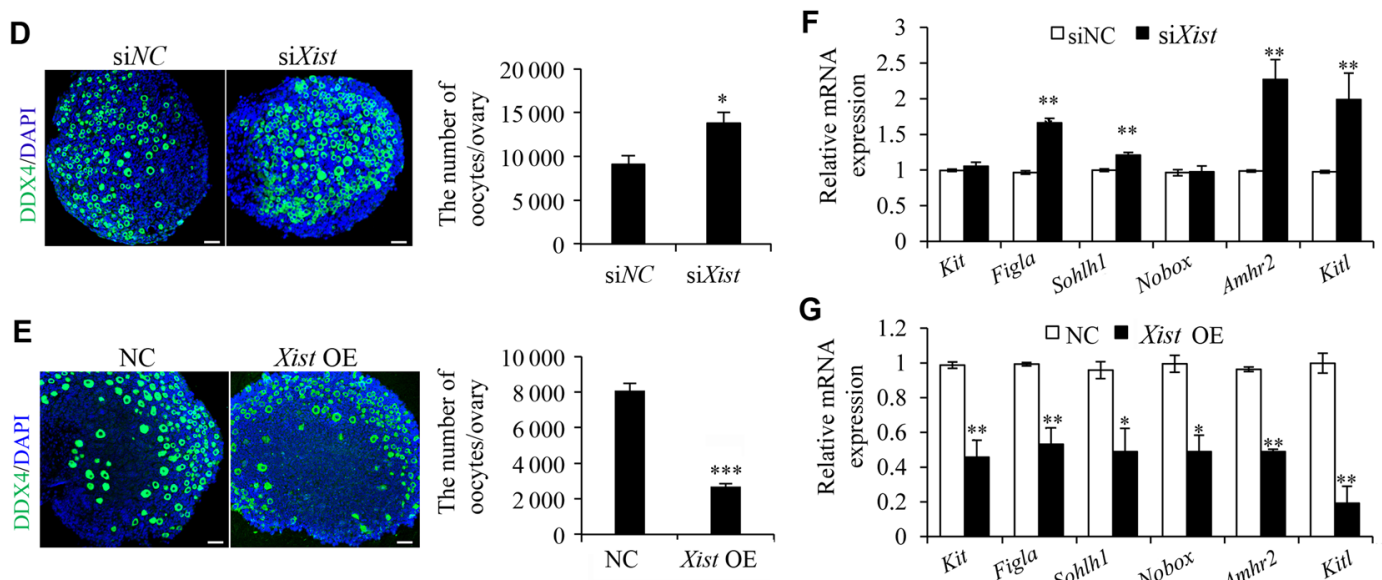

Fig. 1 Xist expression in perinatal ovaries is essential for PF pool formation. a qRT-PCR analysis of Xist expression at the indicated time points. Gapdh was used to normalize samples. b, c qRT-PCR analysis of Xist expression in newborn ovaries transfected with siXist (b), and Xist OE (c), relative to respective controls. $\mathbf{d}$, e Representative images of DDX4 immunofluorescence staining (left) and quantification of follicles (right) in newborn ovaries transfected with siXist (d), and Xist OE (e). Scale bars: $50 \mu \mathrm{m}$. f, g qRT-PCR analysis of genes involved in PF survival and development in siXist (f), and Xist OE (g) transfected ovaries, relative to respective controls. Student's $t$-test: ${ }^{*} P<0.05,{ }^{*} P<0.01,{ }^{* * *} P<0.001$.

membrane vacuoles filled with degraded organelles in Xist-overexpressed oocytes (Fig. 2D), which is the typical appearance of autophagosomes ${ }^{32}$. To further confirm that autophagy is essential for oocyte death under Xist OE overexpression, we calculated the number of oocytes in 0.5 dpp ovaries treated with 3MA. The number of oocytes that survived in the 3MA group was higher than that in the control group, while Xist overexpression partly abrogated this stimulatory effect of 3MA (Fig. 2E). Finally, the $0.5 \mathrm{dpp}$ ovaries transfected with pcDNA-Xist were cultured for $96 \mathrm{~h}$ and then transplanted under the kidney capsule of ovariectomized adult recipient mice (8-wk old) and monitored for $14 \mathrm{~d}$. Ovarian histology analysis revealed a significant loss of activated follicles in the Xistoverexpressed group compared to the controls (Fig. 2F, G). Together, these results suggested that Xist promotes perinatal oocyte loss through oocyte autophagy.

\section{Xist modulates miR-23b-3p and miR-29a-3p maturationin perinatal mouse ovaries}

We next followed upon the underlying mechanism of Xist in regulating oocyte autophagy during perinatal PF formation. Recent studies have demonstrated that nuclear lncRNA regulates miRNA expression by suppressing its maturation process in the nucleus ${ }^{33,34}$. Given that Xist is also a nuclear lncRNA and that miRNAs are essential to promote oocyte survival and decrease apoptosis ${ }^{26-29}$, we wondered whether Xist may also play such a role in regulating oocyte death during PF formation. Previous studies have profiled differentially expressed miRNAs for association with premature ovarian failure development in both animal models and patients, including $m i R-23 b-3 p$ and $m i R-29 a-3 p^{35,36}$. We particularly selected these two miRNAs for further validation since the RNA fragments of the pre-miR-23b and pre-miR-29a are part of the Xist sequences (Fig. 3A), implying that Xist may regulate $m i R$ $23 b-3 p$ and $m i R-29 a-3 p$ expression. Additionally, accumulating studies have demonstrated that both $m i R-23 b$ $3 p$ and $m i R-29 a-3 p$ can regulate autophagy in a wide range of cells ${ }^{37-39}$, which may help raise the possibility that expression of $m i R-23 b-3 p$ and $m i R-29 a-3 p$ modulated by Xist in perinatal mouse ovaries is also associated with perinatal oocyte autophagy. To test this, we first confirmed that pre-miR-23b and pre-miR-29a were enriched in the nuclear fraction of cells from $0.5 \mathrm{dpp}$ ovaries (Fig. 3B and Supplementary Fig. S1). Then, we applied a biotin-avidin pulldown assay to further delineate the direct interaction between Xist and pre-miR-23b and pre$m i R-29 a$ in the nucleus. Briefly, a biotin-labeled-specific anti-pre-miR-23b or anti-pre-miR-29a probe was 

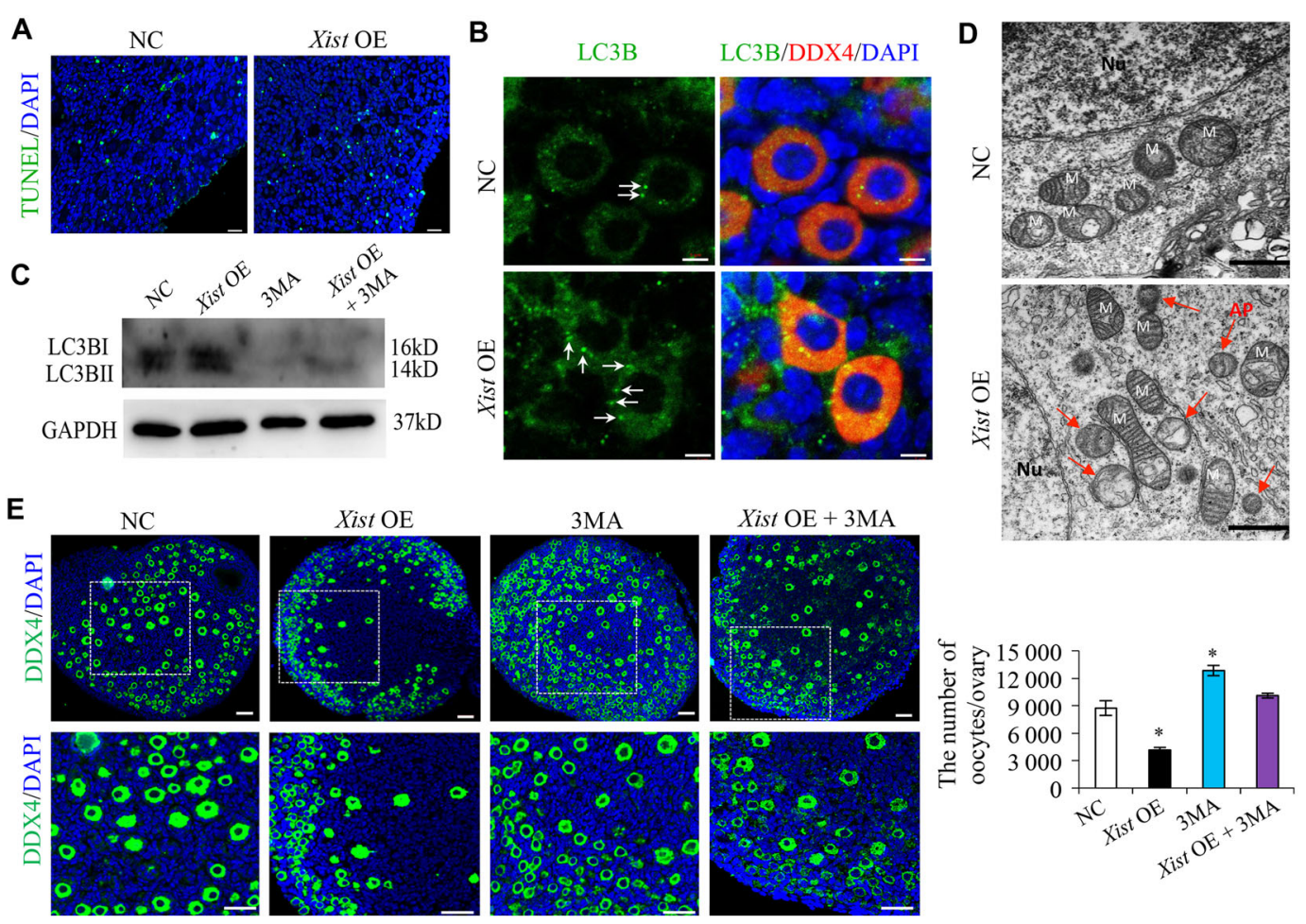
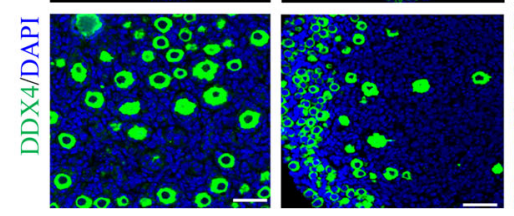

$\mathbf{F}$

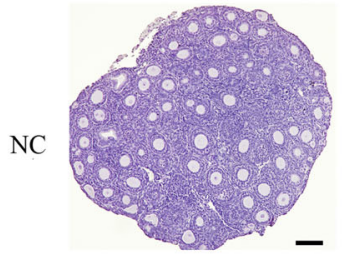

Xist $\mathrm{OE}$

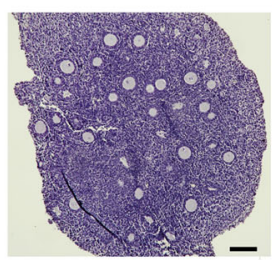

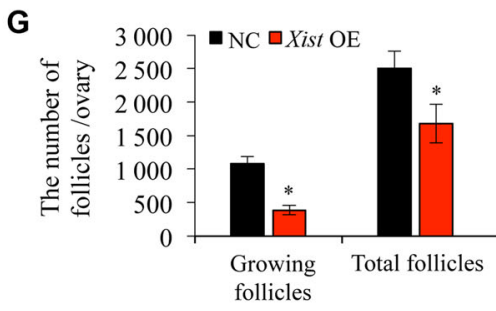

Fig. 2 Xist promotes oocyte autophagy during PF formation. a TUNEL staining in newborn ovaries transfected with Xist-pcDNA3.1 (Xist OE) or the empty vector control (NC). The nucleus was stained by DAPI (blue). Scale bars: $20 \mu \mathrm{m}$. b Immunofluorescence staining of LC3B (green), and DDX4 (red) in newborn ovaries transfected with Xist-OE compared to NC control. Scale bars: $5 \mu \mathrm{m}$. Arrows indicating LC3B puncta. c WB analysis of active LC3B expression in newborn ovaries treated under indicated condition. $\mathbf{d}$ TEM analysis of autophagosomes in newborn ovaries transfected with XistOE compared to NC control. Red arrow indicating autophagosomes. Scale bars: $1 \mu \mathrm{m}$. e Representative images of DDX4 immunofluorescence staining (left) and quantification of follicles (right) in newborn ovaries treated under indicated condition. The nucleus was stained by DAPI (blue). Scale bars: $50 \mu \mathrm{m}$. f, $\mathbf{g}$ Representative images (f) and quantification of follicles $(\mathbf{g})$ in the ovary transfected under indicated condition. Scale bar, $20 \mu \mathrm{m}$. Student's t-test: ${ }^{*} P<0.05$.

synthesized and incubated with nuclear lysate from 3T3 cells. Pre-miR-23b or pre-miR-29a was co-precipitated via avidin-conjugated agarose beads, and the levels of Xist in the pulldown complex were analyzed by qRT-PCR. As shown in Fig. 3C, Xist was significantly enriched in the biotin-labeled pre-miR-23b or pre-miR-29a pulldown product compared to Bio-pre-NC control probe, suggesting that Xist can directly bind to pre-miR-23b or pre$m i R-29 a$ in the nucleus, thus preventing the export of pre$m i R-23 b / p r e-m i R-29 a$ to the cytoplasm. Therefore, when we modulated Xist expression in 3T3 cells, we observed that both pre-miR-23b and pre-miR-29a levels were elevated upon Xist overexpression, and decreased upon Xist knockdown, compared to their respective controls (Fig. 3D). Correspondingly, overexpression of Xist decreased while depletion of Xist increased the expression of mature $m i R-23 b-3 p$ and $m i R-29 a-3 p$ (Fig. 3E). The expression of Xist positively correlated with the expression of pre-miR$23 b$ and pre-miR-29a, but negatively with mature $m i R$ $23 b-3 p$ and $m i R-29 a-3 p$, suggesting that Xist may regulate $m i R-23 b$ and $m i R-29 a$ maturation process.

To assess this hypothesis in vivo, we transfected $0.5 \mathrm{dpp}$ mouse ovaries with pcDNA3.1-Xist or siXist, and found that overexpression of Xist significantly increased the expression of pre-miR-23b and pre-miR-29a, while knockdown of Xist obviously decreased the expression of 
Position: chrX: $103474007-103474028$ (-)
Xist WT: 5'.. GUCAUUCAUUCACAUGGUGCUC.
-miR-29a: 3'.. AUUGGCUAAAGUCUACCACGAU.

C

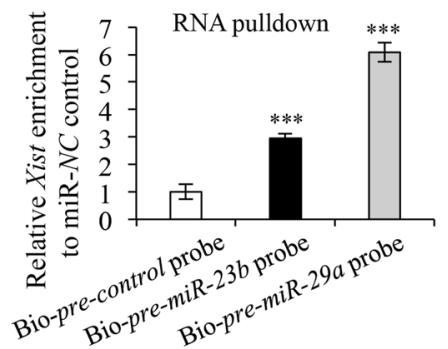

$\mathbf{F}$

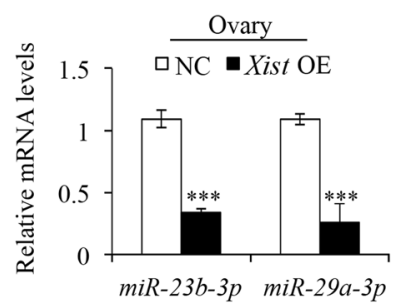

$\mathbf{G}$

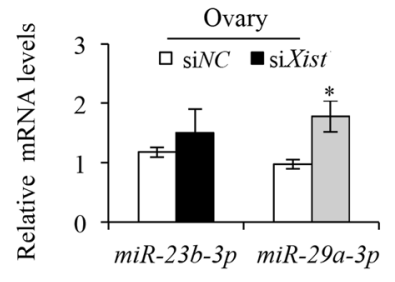

Fig. 3 Xist regulates miR-23b and miR-29a maturation process. a The predicted pre-miR-23b and pre-miR-29a binding sites in the Xist transcript. $\mathbf{b}$ qRT-PCR analysis of Xist, pre-miR-23b, and pre-miR-29a expression in nuclear and cytoplasmic fraction relative to the whole cells from $0.5 \mathrm{dpp}$ ovaries. c Enrichment of Xist pulled down by biotin-pre-miR-23b, biotin-pre-miR-29a, or biotin-pre-miR-NC control in the nuclear fraction from $3 T 3$ cells. $\mathbf{d}$ qRTPCR analysis of Xist, pre-miR-23b, and pre-miR-29a expression in Xist overexpression or Xist knockdown 3T3 cells. e qRT-PCR analysis of miR-23b-3p, and miR-29a-3p expression in Xist overexpression or Xist knockdown $3 T 3$ cells. $\mathbf{f}, \mathbf{g}$ Relative levels of miR-23b-3p and miR-29a-3p in cultured newborn ovaries transfected with Xist or empty control (f), and in ovaries transfected with siXist or scrambled control (g). Student's t-test: ${ }^{*} P<0.05$, ${ }^{* * * P} P<0.001$.

pre-miR-23b and pre-miR-29a in the nuclei (Supplementary Fig. S2). Correspondingly, both $m i R-23 b-3 p$ and miR-29a-3p levels were significantly decreased upon Xist overexpression (Fig. 3F), and upregulated upon Xist knockdown, compared to their respective controls (Fig. 3G). These results indicated that Xist may negatively regulate $m i R-23 b-3 p / m i R-29 a-3 p$ expression in the neonatal mouse ovaries. Together, these data demonstrate that the maturation of $m i R-23 b-3 p$ and $m i R-29 a-3 p$ is associated with the expression of Xist in perinatal ovaries.

\section{MiR-23b-3p/miR-29a-3p are involved in the regulation of PF formation in the perinatal mouse ovaries}

Given Xist negatively regulates mature miR-23b-3p/ $m i R-29 a-3 p$, we speculated to observe an inversed expression pattern of $m i R-23 b-3 p / m i R-29 a-3 p$ in perinatal mouse ovaries to that of Xist. Actually, qRT-PCR analysis just confirmed that $m i R-23 b-3 p / m i R-29 a-3 p$ expression increased significantly along with the establishment of the PF pool from $18.5 \mathrm{dpc}$ to $4.5 \mathrm{dpp}$ (Fig. 4A). In addition, the cytoplasmic localization of the $m i R-23 b-3 p / m i R-29 a-3 p$ by RNA-FISH analysis in oocytes from both cysts and PFs can be readily detected (Fig. 4B). These data raised a possibility that the promoting effect of Xist on oocyte loss during PF formation is likely mediated by its negative regulation on $m i R-23 b-3 p /$ $m i R-29 a-3 p$ expression. To further assess the effect of $m i R-23 b-3 p / m i R-29 a-3 p$ in PF formation, we silenced or overexpressed them in $0.5 \mathrm{dpp}$ ovaries, respectively. The silencing or overexpression efficiency was then confirmed $96 \mathrm{~h}$ post transfection (Fig. 4C, D). Then, the cultured ovaries were subjected to histological examination. Immunohistochemistry analysis with anti-DDX4 antibody showed that anti-miR-23b-3p or anti-miR-29a-3p significantly decreased oocyte number compared to the controls (Fig. 4E), and overexpression of $m i R-23 b-3 p$ or $m i R-29 a-3 p$ exhibited more oocytes than the miRNA controls $96 \mathrm{~h}$ post transfection (Fig. 4F). Analysis of LC3B 
A

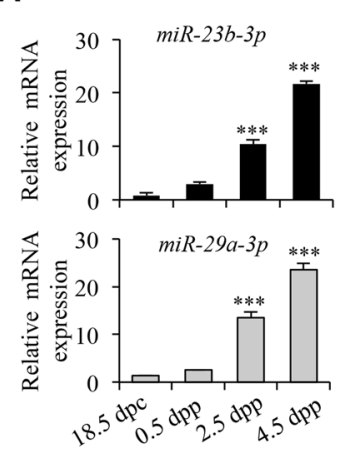

D
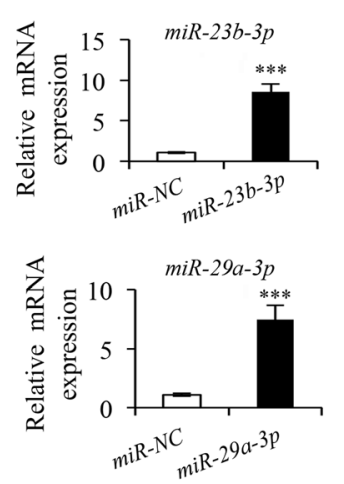

G

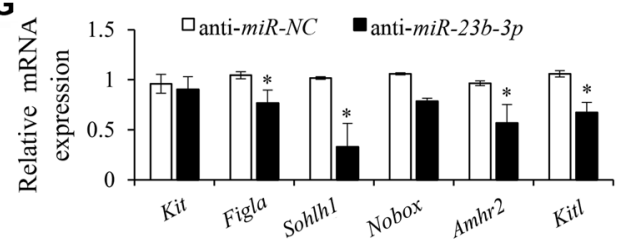

I

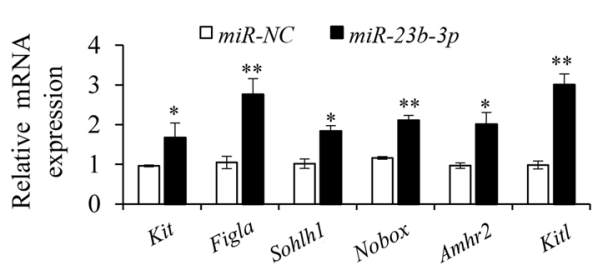

B

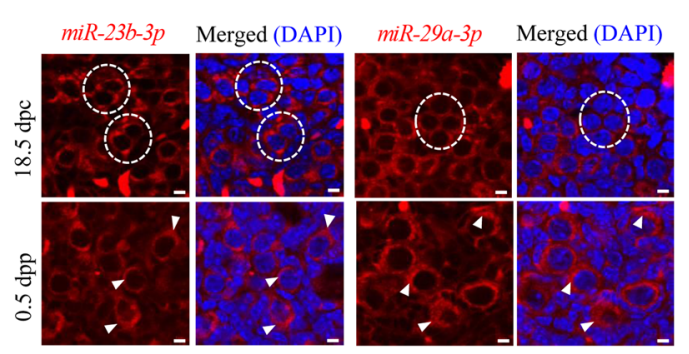

E

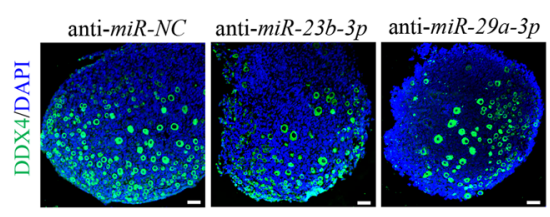

F

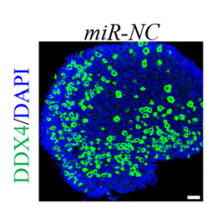

$m i R-23 b-3 p$

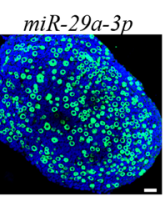

C
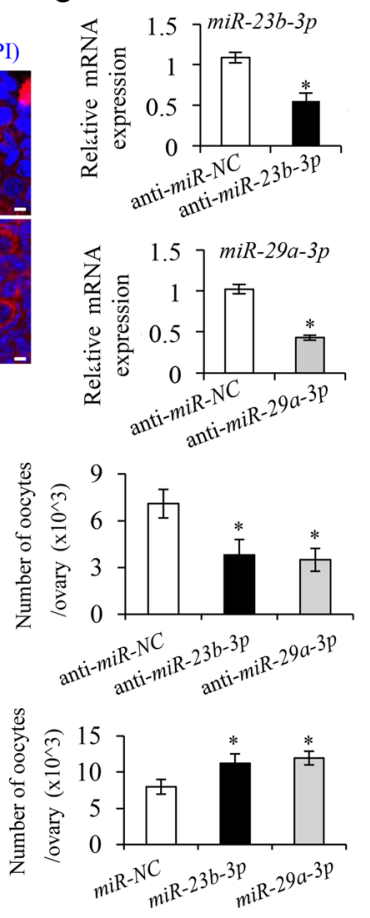

H

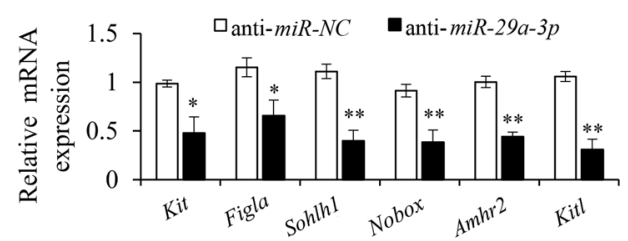

J

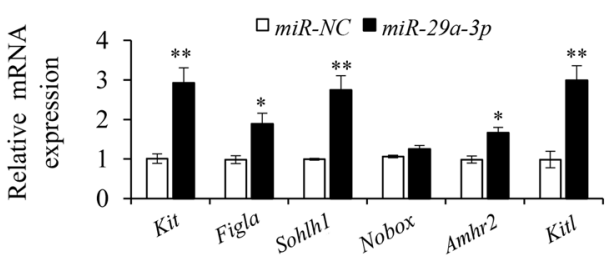

Fig. 4 Expression of miR-23b-3p and miR-29a-3p are essential for PF formation in the perinatal mouse ovaries. a qRT-PCR analysis of miR-23b$3 p$ (top) and miR-29a-3p (bottom) expression at the indicated time points. Gapdh was used to normalize samples. b RNA-FISH analysis of miR-23b-3p and miR-29a-3p expression in perinatal mouse ovaries from $18.5 \mathrm{dpc}$, and $0.5 \mathrm{dpp}$. The nucleus was stained by DAPI (blue). Dashed circle indicating representative cysts. Arrows heads indicating oocytes. Scale bars: $5 \mu$ m. c, d qRT-PCR analysis of miR-23b-3p (top) and miR-29a-3p (bottom) expression in newborn ovaries transfected with anti-miR-23b-3p, anti-miR-29a-3p, or anti-miR-NC (c), and mimics for miR-23b-3p, miR-29a-3p, or miR-NC (d). e, $\mathbf{f}$ Representative DDX4 immunofluorescence images (left) and quantification of follicles (right) in newborn ovaries transfected with anti-miR-23b-3p, anti-miR-29a-3p, or anti-miR-NC (e), and mimics for miR-23b-3p, miR-29a-3p, or miR-NC (f). Scale bars: $50 \mu \mathrm{m}$. $\mathbf{g}-\mathbf{j}$ qRT-PCR analysis of genes involved in PF survival and development in newborn ovaries transfected with anti-miR-23b-3p (g), anti-miR-29a-3p (h), or anti-miR-NC, and mimics for miR-23b-3p (i), miR-29a-3p (j), or miR-NC. Student's $t$-test: ${ }^{*} P<0.05$, ${ }^{* *} P<0.01$, ${ }^{* *} P<0.001$.

expression confirmed that inhibiting $m i R-23 b-3 p$ or $m i R$ $29 a-3 p$ in the newborn ovaries obviously elevated, while overexpression of $m i R-23 b-3 p$ or $m i R-29 a-3 p$ decreased oocyte autophagy compared to their respective controls (Supplementary Fig. S3). Furthermore, expression of the genes related to PF formation decreased in anti-miR-23b-
$3 p$ or anti-miR-29a-3p treated ovaries (Fig. $4 \mathrm{G}, \mathrm{H}$ ), while increased in $m i R-23 b-3 p$ or $m i R-29 a-3 p$ overexpressed group (Fig. 4I, J). These results indicated that $m i R-23 b-3 p /$ $m i R-29 a-3 p$ have the opposite effect to that of Xist, which may promote PF formation in perinatal mouse ovaries, possibly by preventing oocyte loss. 


\section{Xist relieves the inhibition of miR-23b-3p/miR-29a-3p on their common target STX17}

To find out downstream genes sharing the regulatory role of $m i R-23 b-3 p / m i R-29 a-3 p$ with Xist, we used five online bioinformatic tools (TargetScan, miRDB, miRWalk, Starbase, and RNA22) to predict the potential cotarget genes of miR-23b-3p/miR-29a-3p (Fig. 5A). Among the seven co-target genes with sequence complementarity to both $m i R-23 b-3 p / m i R-29 a-3 p$, STX17 protein has attracted our particular attention, since it is directly implicated in the autophagy process ${ }^{40}$. Syntaxin 17 (STX17) is a soluble N-ethylmaleimide-sensitive factor attachment protein receptor (SNARE) protein that drives the maturation of autophagosomes acquiring degradative enzymes by fusing with the lysosome ${ }^{41,42}$. STX17 localizes to the outer membrane of autophagosomes, and binds directly to the autophagosomal protein, $\mathrm{LC}^{43}$. Failure of autophagosome-lysosome binding leads to the blockade of the autophagic flux and abnormal degradation of autophagosomes ${ }^{44}$. Therefore, STX17 may function as the downstream target for $m i R-23 b-3 p / m i R-29 a-3 p$ in regulating oocyte autophagy in the perinatal ovaries, and thus was selected for further analysis. We first synthesized wild-type (WT) and mutant Stx17 3'UTR (Mut1 corresponding to $m i R-23 b-3 p$, and Mut2 to $m i R-29 a-3 p$ ), and constructed them into luciferase reporters, separately (Fig. $5 B)$. Luciferase reporter assays demonstrated that overexpression of $m i R-23 b-3 p$ or $m i R-29 a-3 p$ repressed luciferase activity in HEK293 cells transfected with WT Stx17 $3^{\prime}$ UTR reporter plasmid, whereas such effect was not observed in the mutant reporters (Fig. 5C). Next, biotinlabeled miRNA pulldown assays verified that $S t x 17$ is the target gene for $m i R-23 b-3 p / m i R-29 a-3 p$ (Fig. 5D). Further analyses revealed that STX17 expression could be downregulated by overexpression of $m i R-23 b-3 p$ or $m i R$ $29 a-3 p$, while upregulated by inhibitors for $m i R-23 b-3 p$ or $m i R-29 a-3 p$ (Fig. 5E, F). Since Xist inhibits the maturation of $m i R-23 b-3 p / m i R-29 a-3 p$, upregulation of Xist positively affected STX17 expression through modulating $m i R-23 b-3 p / m i R-29 a-3 p$ (Fig. 5G), while an opposite effect was observed upon Xist inhibition (Fig. 5H). Together, these findings confirmed the existence of a Xist$m i R-23 b-3 p / m i R-29 a-3 p-S T X 17$ regulatory axis in the perinatal ovaries.

\section{STX17 is responsible for Xist-mediated perinatal oocyte loss}

To further verify STX17 is the downstream effector in Xist-miR-23b-3p/miR-29a-3p-STX17 regulatory axis in regulating perinatal oocyte loss, we first showed a similar expression pattern of STX17 to that of Xist in the perinatal mouse ovaries (Fig. 5I). Next, silencing STX17 in 0.5 dpp ovaries significantly increased the oocyte number compared to controls, after $96 \mathrm{~h}$ of in vitro culture (Fig. 5J,
K). Additionally, expression of the genes related to PF formation also increased in siStx17-treated ovaries (Fig. 5L). Furthermore, STX17-silenced neonatal ovaries showed a suppressed expression pattern of LC3B (Fig. 5M and Supplementary Fig. S4A). Conversely, we also overexpressed STX17 in the neonatal ovaries (Supplementary Fig. S4B), and observed that STX17 overexpression significantly decreased the oocyte number compared to controls after $96 \mathrm{~h}$ of in vitro culture (Supplementary Fig. S4C). Meanwhile, STX17-overexpressed neonatal ovaries showed an increased expression pattern of LC3B (Supplementary Fig. S4D). These data suggested that STX17 is involved in regulating PF pool formation through accelerating oocyte autophagy. Finally, to verify the ability of Xist to promote perinatal oocyte loss in an STX17dependent manner, we treated $0.5 \mathrm{dpp}$ ovaries with pcDNA3.1-Xist, siStx17, or both in combination. The results demonstrated that knockdown of STX17 partially rescued the suppressive effects of Xist on PF pool formation (Fig. 5N-P).

\section{YY1 is essential for Xist transcription during PF pool formation}

It is essential for transcription factor Yin-Yang 1 (YY1) to bind directly to Xist promoter and activate Xist transcription in multiple cells ${ }^{45,46}$. To test whether YY1 facilitates Xist expression in the perinatal ovaries, we first performed ChIP assays using YY1 antibody and detected YY1 binding at Xist promoter with qPCR primers flanking the YY1 binding sites (Fig. 6A). We observed a strong enrichment of YY1 to this region in 0.5 dpp ovaries, while YY1 binding dramatically decreased in $4.5 \mathrm{dpp}$ ovaries (Fig. 6A). This binding pattern was closely related to a rapid decrease of Xist expression from $18.5 \mathrm{dpc}$ to $4.5 \mathrm{dpp}$ ovaries, suggesting that YY1 binding may positively regulate Xist expression in the perinatal ovaries. Next, we examined the expression levels of YY1 during PF formation, and found a gradual decrease of YY1 expression in a time-specific manner from $18.5 \mathrm{dpc}$ to $4.5 \mathrm{dpp}$ (Fig. 6B, C). Given previous studies have shown that depletion of YY1 specifically inhibits Xist expression in other female cells $^{45,46}$, our data raised a possibility that the decreased YY1 in perinatal ovaries may contribute to the downregulated Xist. To confirm this causal relationship between YY1 and Xist expression, gain or loss of function experiments were performed in $0.5 \mathrm{dpp}$ ovaries by silencing or overexpressing YY1. The qRT-PCR and WB analyses showed that si $Y y 1$ transfection decreased, and pcDNA-Yy1 transfection increased YY1 expression in 4.5 dpp ovaries (Fig. 6D, E). YY1 suppression significantly inhibited Xist expression, while overexpression of YY1 increased Xist expression (Fig. 6F). As a transactivator of Xist, YY1 was shown to play a similar role during PF pool formation to that of Xist, in an STX17-dependent manner 


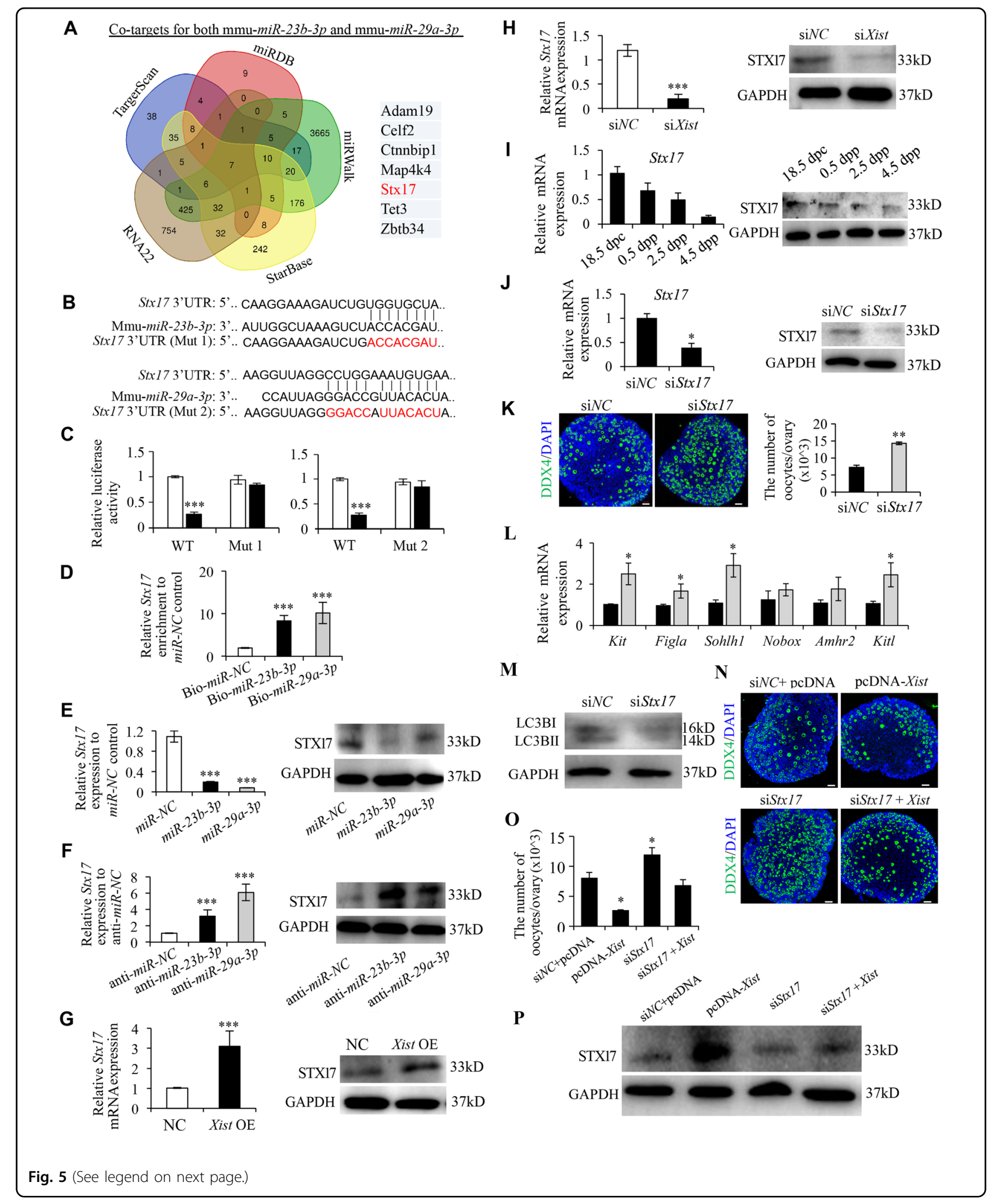


(see figure on previous page)

Fig. 5 STX17 is a downstream target of Xist-miR-23b-3p/miR-29a-3p axis and regulates PF formation. a The Venn diagram shows overlapping of the co-target genes of miR-23b-3p/miR-29a-3p from five representative databases. $\mathbf{b}$ The predicted binding sites of miR-23b-3p and miR-29a-3p binding sites in the $3^{\prime}$-UTR of Stx17. The red nucleotides represent mutant sequences of target sites. c Luciferase activities in HEK293 cells transfected with WT or mutant Stx17 plasmid together with miR-23b-3p or miR-29a-3p, or miR-NC. d Enrichment of Stx 17 pulled down by biotin-miR-23b-3p, biotin-miR-29a-3, or biotin-miR-NC control in 3 T3 cells. e-h Relative Stx17 mRNA (left), and STX17 protein (right) expression in cultured newborn ovaries transfected with mimics for miR-23b-3p, miR-29a-3p, or miR-NC (e), anti-miR-23b-3p, anti-miR-29a-3p, or anti-miR-NC (f), Xist or empty control (g), and siXist or scrambled control (h). i Relative StX17 mRNA (left), and STX17 protein (right) expression at the indicated time points. $\mathbf{j}$ Relative Stx 17 mRNA (left), and STX17 protein (right) expression in cultured newborn ovaries transfected with siStx17, or siNC as a control. k Representative images (left) and quantification of follicles (right) in newborn ovaries transfected with siStx17, or siNC. Scale bars: $50 \mu \mathrm{m}$. I qRT-PCR analysis of genes involved in PF survival and development in newborn ovaries transfected with siStx17, and siNC as a control. $\mathbf{m}$ WB analysis of LC3B expression in newborn ovaries treated under indicated condition. $\mathbf{n}$ Representative images of DDX4 in newborn ovaries co-transfected with pcDNA-Xist or empty vector together with siStx 17 or scrambled control. Scale bars: $50 \mu \mathrm{m}$. o Quantification of follicles in $\mathbf{n}$. $\mathbf{p}$ WB analysis of STX17 expression in newborn ovaries treated under indicated condition. GAPDH was used as a loading control. Student's $t$-test: ${ }^{*} P<0.05,{ }^{* *} P<0.01,{ }^{* * *} P<0.001$.

(Fig. 6G-I). In agreement with the previous studies showing that YY1 is implicated in autophagy in multiple cells $^{47,48}$, we then confirmed that YY1 positively regulated the LC3B protein expression in the newborn ovaries (Supplementary Fig. S5A). As we showed that Xist promotes perinatal oocyte loss through autophagy in an STX17-dependent manner (Figs. 2 and 5), YY1 overexpression also rescued the decreased LC3B expression mediated by STX17 depletion (Supplementary Fig. S5B). These data supported the existence of a YY1-Xist-miR$23 b-3 p / m i R-29 a-3 p-S T X 17$ regulatory axis during PF formation.

\section{Discussion}

It is well accepted that cellular apoptosis and autophagy processes are responsible for programmed oocyte loss occurring briefly around the time of birth $^{5,31,40}$. Meanwhile, lncRNAs are emerging as central regulators in controlling cell autophagy or apoptosis in various diseases ${ }^{49,50}$. However, there is no such lncRNA report in the process of PF formation. Here, we demonstrated that Xist promotes perinatal oocyte loss mainly through oocyte autophagy. Mechanically, we showed explicitly that, during the early period of PF pool formation, YY1 activates Xist transcription; Xist blocks miR-23b-3a/miR-29a-3a biogenesis process, resulting in decreased mature $m i R-23 b-3 a / m i R-29 a-3 a$ expression; Given STX17 is a co-target for $m i R-23 b$ $3 a / m i R-29 a-3 a$ and that STX17 is a critical factor in the process of autophagy, Xist relieving the inhibition of $m i R-23 b-3 p / m i R-29 a-3 p$ on STX17 finally leads to an increased STX17 expression, which promotes massive oocyte loss through oocyte autophagy (Fig. 6J). These findings indicate that Xist is an indispensable primordial folliculogenesis factor that controls PF pool formation.

Xist, whose gene product is a lncRNA, is exclusively expressed on the inactivated $\mathrm{X}$-chromosome and its abnormal expression has been linked strongly to the process of X-chromosome inactivation ${ }^{51,52}$. It is speculated that variation in expression of Xist gene that leads to a preferential silencing of genes on the X-chromosome related to the maintenance of ovarian function may consider being a susceptibility factor for $\mathrm{POI}^{52}$. Clinically, the inadequate number of follicles in the perinatally generated PF pool is one of the causative factors for POI, leading to the shortened reproductive life span. But whether Xist is involved in PF pool formation is unclear. The only available clue is coming from the transcriptome analysis of IncRNA expression in human primordial, primary and small antral follicles, with Xist being identified as one of highly expressed $\operatorname{lncRNAs}{ }^{15}$. Our finding that interference of Xist expression affects the oocyte loss during the formation of PF pool may lend support to this hypothesis and help better understand the novel functional relationship between Xist and pathogenesis of POI.

Accumulating studies have demonstrated that, in addition to protein-mediated post-transcriptional control in miRNA biogenesis, nuclear ncRNAs can also function as negative regulators for miRNA biogenesis as miRNA maturation process begins in the nucleus. For example, colon cancer-associated transcript-2 (Ccat2), a lncRNA mainly located in the nucleus, has been reported to selectively block miR-145 maturation by inhibiting premiR-145 export to the cytoplasm in colon cancer cells ${ }^{33}$. Tang et al. ${ }^{53}$ reported that, in the cell nucleus, miR-709 directly binds to pri-miR-15a/16-1 and prevents its processing into pre-miR-15a/16-1, leading to a suppression of miR-15a/16-1 biogenesis. In line with these reports, our findings that expression of Xist in the perinatal mouse ovaries positively correlated with expression of pre-miR$23 b /$ pre-miR-29a, but inversely correlated with mature $m i R-23 b-3 a / m i R-29 a-3 a$, and that Xist binds to the pre$m i R-23 b /$ pre-miR-29a in the nucleus, imply that Xist may block the export of pre-miR-23b/pre-miR-29a to the cytoplasm, resulting in decreased mature miR-23b-3a/ $m i R-29 a-3$. In the process of miRNA biogenesis, exportin$5^{54}$, and exportin- $1^{55}$ are identified to be nuclear export 
A

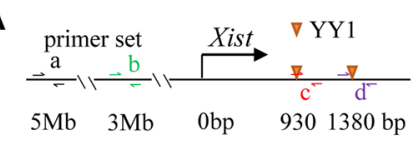

YY1 ChIP

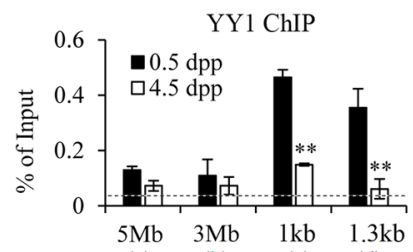

primer set (a) (b)

B

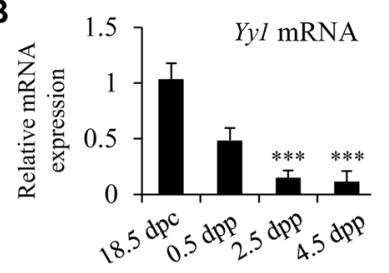

C

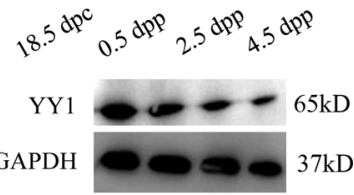

D

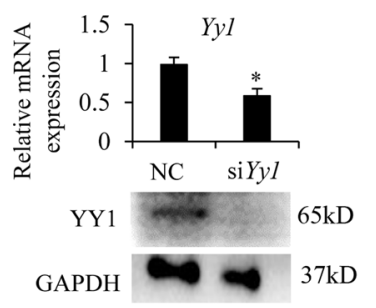

E

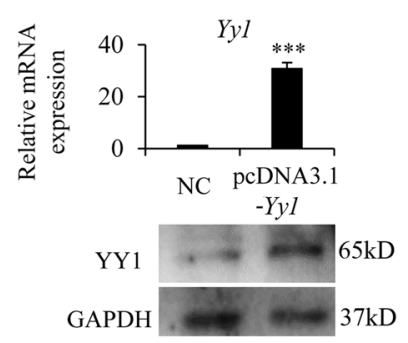

F
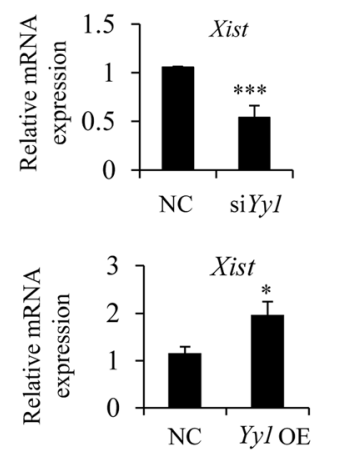

G
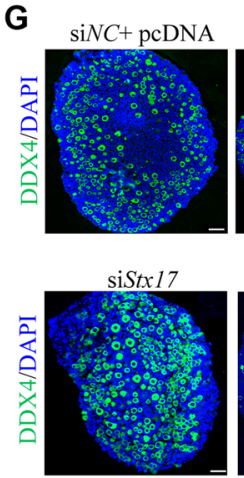

$\mathrm{kD}$

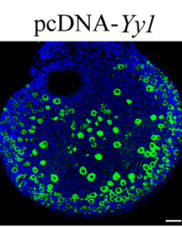

$\operatorname{siStx} 17+Y y 1$

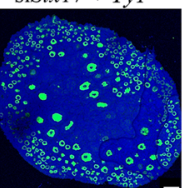

H
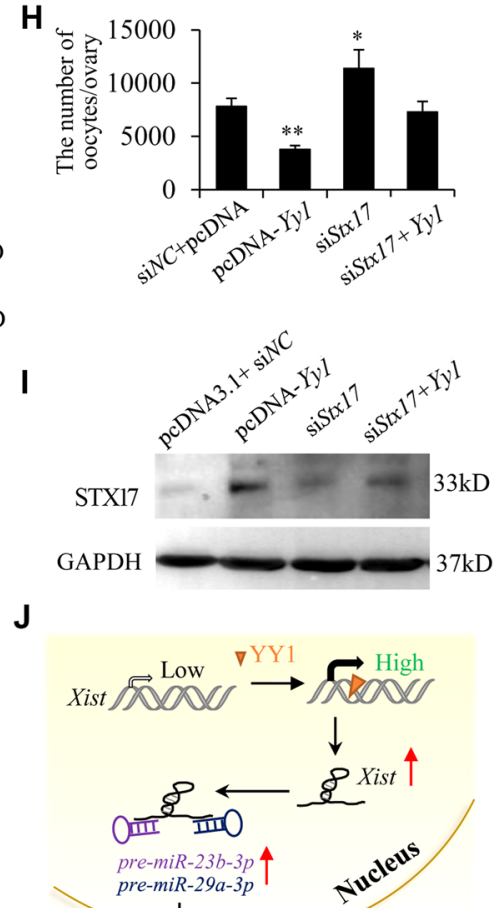

pre-miR-29a-3p
pre-

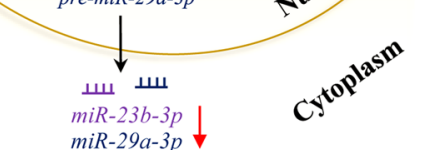

miR-23b-3p

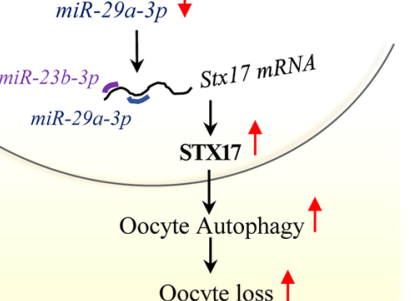

Fig. 6 YY1 is essential for Xist expression and functions as an upstream regulator for Xist-miR-23b-3p/miR-29a-3p-Stx17 axis during PF formation. a Schematic representation for $Y Y 1$ binding motif within the proximal promoter region of Xist, with respective primer sets to amplify $Y Y 1$ binding region. The upstream control region is also represented (top). Bottom: ChIP analysis of YY1 binding in newborn mouse ovaries at 0.5 dpp, and $4.5 \mathrm{dpp}$. The dashed line represents the basal lgG binding. b, c Relative Yyl mRNA (b), and YY1 protein (c) expression in perinatal ovaries at indicted time. d, e Relative Yy1 mRNA (top), and YY1 protein (bottom) expression in cultured newborn ovaries transfected with siYy1 or control (d), pcDNA3.1-Yyl, and NC control (e). f Relative Xist mRNA expression in cultured newborn ovaries transfected with siYy1 or siRNA control (top), pcDNA3.1-Yy1 or NC control (bottom). $\mathbf{g}, \mathbf{h}$ Representative DDX4 immunofluorescence images (g) and quantification of follicles (h) in newborn ovaries transfected under indicated condition. Scale bars: $50 \mu \mathrm{m}$. i WB analysis of STX17 expression in newborn ovaries treated under indicated condition. j Proposed model for Xist-miR-23b-3p/miR-29a-3p-STX17 axis in regulating oocyte loss in the perinatal mouse ovaries. Student's $t$-test: * $P<$ $0.05,{ }^{* *} P<0.01,{ }^{* * *} P<0.001$.

factors to transport pre-miRNAs from the nucleus to the cytoplasm. It is not clear whether Xist affects the function of either exportin-5 or exportin-1 and this requires further investigation. Additionally, recent studies found that Xist functions as a competing endogenous RNA (ceRNA) to sponge the common miRNAs, and therefore insulates the miRNAs and facilitates the corresponding miRNAtargeted transcripts in tumorigenesis ${ }^{56,57}$, or in the regulation of inflammation and apoptosis ${ }^{58}$. Together, these studies significantly expand the biological function of
Xist/miRNA/mRNA axis in regulating a broad spectrum of biological processes.

Transcriptional regulation of Xist has been widely investigated and several transcription factors are identified to be required for its transcription ${ }^{51}$. A recent study has carried out in silico analysis of Xist promoter regions in seven species of eutherian mammals and revealed clustered YY1 consensus binding sites evolutionarily well conserved in all species tested, including human and mouse. Furthermore, YY1 can directly bind to the Xist 
promoter region and promote Xist expression during initiation and maintenance of X-chromosome inactivation, suggesting that $\mathrm{YY} 1$ binding triggers the activity of the Xist promoter ${ }^{46}$. In consistence with this activity of YY1, our study in the perinatal ovaries also verified the binding of YY1 at Xist proximal promoter region, which is necessary for Xist transactivation during PF pool formation. Additionally, our findings that the same expression pattern of YY1 as that of Xist was observed in the perinatal ovaries, and that modulation of YY1 expression induced a similar phenotype during PF formation as that of Xist, strongly suggest that YY1 may function as an important upstream regulator in Xist-miR-23b-3p/miR$29 a-3 p$ axis.

Collectively, we elucidate a novel mechanism by which lncRNA Xist, activated by transcription factor YY1, blocks $m i R-23 b-3 p / m i R-29 a-3 p$ maturation process by inhibiting pre-miR-23b/pre-miR-29a export to the cytoplasm, and relieves the inhibition of $m i R-23 b-3 p / m i R-29 a-3 p$ on STX17, resulting in massive oocyte loss through oocyte autophagy in the perinatal ovaries. Our work may offer new insights into the mysteries of early folliculogenesis in mammalian ovaries, and highlight the potential clinical value of Xist-miR-23b-3p/miR-29a-3p-STX17 axis in the diagnosis and treatment of POI.

\section{Materials and methods}

\section{Mice and ovary culture}

All wild-type mice were purchased from the Animal Core Facility of Nanjing Medical University. The Animal Care and Use Committee of Nanjing Medical University approved all the animal experiments. The mice were housed under a 12/12-h dark/light cycle with free access to food and water at $20-22^{\circ} \mathrm{C}$. Mice were mated using timed mating, and the presence of a vaginal plug was defined as $0.5 \mathrm{dpp}$. Embryonic ovaries were collected at $18.5 \mathrm{dpc}$, and neonatal ovaries were collected at $0.5,2.5$, and $4.5 \mathrm{dpp}$. For ovary culture, 4 ovaries were randomly selected and placed in a 24-well dish and cultured in DMEM/F-12 medium (Gibco, Life Technologies, NY, USA) supplemented with penicillin-streptomycin and ITS at $37^{\circ} \mathrm{C}$ under $5 \% \mathrm{CO}_{2}$. The $m i R-23 b-3 p / m i R-29 a-3 p$ mimics, inhibitors, and their corresponding scramble controls were ordered from GenePharma (Shanghai, China). The Xist, and $Y y 1$ overexpression plasmids, siRNAs against Xist, and $Y y 1$ were from General Biosystems (Anhui, China). The oligonucleotide sequences were listed in Supplementary Table 1. For ovaried treated with $2.5 \mathrm{mM}$ of 3MA (S2767, Selleck, USA), dimethylsulfoxide (DMSO) was used as a control.

\section{Cell culture}

Human embryonic kidney (HEK) 293 cells and mouse NIH/3T3 cells were purchased from the Cell Resource
Center of the Shanghai Institute for Biological Sciences (Shanghai, China). Both cell types cultured in Dulbecco's Modified Eagle's medium (DMEM; HyClone, USA) supplemented with $10 \%$ fetal bovine serum (FBS; Gibco, USA) and $1 \%$ penicillin/streptomycin (P/S; Thermo Fisher Scientific, Rockford, IL, USA) and were incubated in a humidified incubator at $37^{\circ} \mathrm{C}$ with $5 \% \mathrm{CO}_{2}$.

\section{Quantitative real-time PCR}

RNA was extracted from mouse ovaries using TRIzol (\#15596026, Invitrogen, MA, USA), followed by reverse transcription with First Strand cDNA Synthesis Kit (K1621, Thermo Scientific, MA, USA), and qRT-PCR analysis with AceQ qPCR SYBR Green Master Mix (Q141-02, Vazyme, Jiangsu, China). The primers are summarized in Supplementary Table 2. The relative fold change of gene expression was calculated using the relative standard curve method $\left(2^{-\Delta \Delta C t}\right)$.

\section{Isolation of nuclear and cytoplasmic fractions}

Cells were washed three times with cold PBS, followed by centrifugation at $500 \mathrm{rpm}$ for $5 \mathrm{~min}$ at $4{ }^{\circ} \mathrm{C}$. Cell pellets were then resuspended in $1 \times$ Hypotonic Buffer $(20 \mathrm{mM}$ Tris, $\mathrm{pH} 8.0 ; 10 \mathrm{mM} \mathrm{NaCl}, 3 \mathrm{mM} \mathrm{MgCl}$, and 10\% NP-40), and incubated on ice for $15 \mathrm{~min}$. After centrifugation at $3000 \mathrm{rpm}$ for $10 \mathrm{~min}$ at $4{ }^{\circ} \mathrm{C}$. the supernatant portion (cytoplasmic extract) was transferred into the precooled tube. The pellet is the crude nuclear fraction. Nuclear pellets were then washed twice with cold PBS.

\section{Western blotting}

Ovaries were lysed with RIPA buffer (CW2333, CWBIO, Beijing, China) with $1 \times$ protease inhibitor (CW2200, CWBIO). The supernatant was collected and about $30 \mu \mathrm{g}$ of denatured protein was separated on a $10 \%$ SDS-PAGE gel, and transferred to a nitrocellulose membrane. The blots were rinsed in TBS containing $0.5 \%$ Tween- 20 and blocked with $5 \%$ nonfat dry milk. After incubation with the primary antibodies overnight at $4{ }^{\circ} \mathrm{C}$, including anti-LC3B (\#2775, Cell Signaling Technology, 1:1000), anti-STX17 (\#31261, Cell Signaling Technology, 1:1000), anti-YY1 (\#22156-1-AP, Proteintech, 1:1000), anti-Lamin B1 (\#12586, Cell Signaling Technology, 1:1000), anti-GAPDH (ab8245, Abcam, 1:2000), and antiFLAG (\#14793, Cell Signaling Technology, 1:1000), the blots were then washed and incubated with corresponding peroxidase-conjugated secondary antibody for $1 \mathrm{~h}$ at room temperature. The signals were visualized using an Enhanced Chemiluminescence Detection Kit (\#32106, Thermo Scientific) on a Bio-Rad gel imaging system.

\section{Luciferase reporter assay}

The wild-type or mutant Xist fragment containing the predicted binding sites of pre-miR-23b or pre-miR-29a 
was cloned into the modified pGL3 luciferase reporter vector (gift from Dr. Chun Lu from Nanjing Medical University). Luciferase reporter plasmid and $40 \mathrm{nM}$ of premiR-23b, pre-miR-29a-3p, or pre-control were cotransfected into HEK293 cells. The luciferase reporter assay system (E2920, Promega) was used to measure luciferase activity.

\section{RNA fluorescent in situ hybridization (RNA-FISH)}

5'-FAM-labeled miR-23b-3p or miR-29a-3p probes were designed and synthesized by General Biosystems (Anhui, China). Hybridizations were carried out using FISH Kit from GenePharma (Shanghai, China). Briefly, the ovaries were fixed in $4 \%$ paraformaldehyde and processed for serial paraffin sectioning at $5 \mu \mathrm{m}$ thickness. After permeabilization, sections were hybridized with specific probes, and 6-diamidino-2-phenylindole (DAPI) was used to stain nuclei. All fluorescence images were captured using Point Scanning Laser Confocal Microscope (ZEISS, Germany).

\section{Biotin-labeled miRNA pulldown assay}

Briefly, the $3^{\prime}$-end biotinylated pre-miR-23b-3p, pre$m i R-29 a-3 p$, or pre-miRNA control probe $(50 \mathrm{nM}$, General Biosystems, Anhui, China) was transfected into 3T3 cell, and then cell nuclear fraction was extracted $48 \mathrm{~h}$ post transfection. Dynabeads MyOne Streptavidin C1 kit (Invitrogen) was used to enrich the biotincoupled RNA complex. The beads were then pelleted and washed to remove unbound materials. Beadsbound RNA was extracted with TRIzol reagent. The abundance of Xist in the isolated fractions was tested by qRT-PCR.

\section{Immunofluorescence}

Sections were deparaffinized, rehydrated, followed by antigen retrieval by boiling the sections in $0.01 \mathrm{M}$ citrate buffer, $\mathrm{pH} 6.0$ for $15 \mathrm{~min}$. Then the sections were blocked in $10 \%$ normal goat serum, and incubated with primary antibodies overnight at $4{ }^{\circ} \mathrm{C}$, including anti-DDX4 (Ab13840, Abcam, 1:200), anti-DDX4(Ab27591, Abcam, 1:200), and anti-LC3B (\#2775, Cell Signaling Technology, 1:100). After 5 washes with PBS, the sections were incubated with secondary antibodies for $1 \mathrm{~h}$ at room temperature. DAPI was used to stain nuclei.

\section{Chromatin immunoprecipitation (ChIP)}

MAGNA ChIP kit (\#17371RF, Millipore, USA) was used to perform the ChIP assay. Cross-linked chromatin from mouse ovaries was prepared and immunoprecipitations were followed with primary antibodies, including antiAgo2 (\#2897, Cell Signaling Technology, $4 \mu \mathrm{g} / \mathrm{sample})$, anti-YY1 (\#22156-1-AP, Proteintech, $4 \mu \mathrm{g} / \mathrm{sample})$ or rabbit IgG (\#12-370, Millipore, $4 \mu \mathrm{g} / \mathrm{sample})$. The enriched chromatin DNA was quantified by qPCR. Primers used are listed in Supplementary Table 2.

\section{TUNEL assay}

Oocyte apoptosis was measured by TUNEL staining with a TUNEL Apoptosis Detection Kit (FITC) (Yeasen, Shanghai, China) on ovary sections. Nuclei DNA was stained with DAPI. The images were photographed using a Carl Zeiss (Oberkochen, Germany) lens.

\section{Transmission electron microscopy (TEM)}

Ovaries were fixed in $2.5 \%$ glutaraldehyde in $0.2 \mathrm{M}$ PBS ( $\mathrm{pH}=7.2$ ) overnight at $4{ }^{\circ} \mathrm{C}$, and processed and wrapped in epoxypropane resin following standard TEM procedures.

\section{Histological evaluation of follicle numbers}

Theo varies were fixed, embedded in paraffin, and serially sectioned at a thickness of $5 \mu \mathrm{m}$. After staining, follicles were counted in every fifth section. To avoid duplicate counts, the only oocyte with a visible nucleus was counted. Germ cells not surrounded by GCs were scored as unassembled cysts. Germ cells surrounded by GCs or a mixture of squamous and cuboidal somatic cells were scored as primordial follicles. The total number of oocytes in each ovary was calculated by multiplication by 5 .

\section{Statistical analysis}

All experiments were repeated at least three independent biological replicates and presented as the mean \pm the standard error of the mean. Statistical analysis was performed using Student's $t$-tests or one-way ANOVA to compare the difference. A value of $P<0.05$ was considered statistically significant.

\section{Author contributions \\ M.Z., X.L., and X.Z. designed the research. M.Z., Q.E., Y.S., S.L., and X.Z. performed the research. M.Z., X.L., and X.Z. analyzed the data and wrote the manuscript. All authors have seen and approved the final version. \\ Funding \\ This work was supported by the National Key Research and Development Program of China [2018YFC1003703, 2018YFC1004203], and NMU Science and Technology Innovation Project [2017NJMUCX007].}

Ethics statement

All animal experiments were approved by the Animal Care and Use Committee of Nanjing Medical University.

Conflict of interest

The authors declare no competing interests.

Publisher's note

Springer Nature remains neutral with regard to jurisdictional claims in published maps and institutional affiliations. 
Supplementary information The online version contains supplementary material available at https://doi.org/10.1038/s41419-021-03831-4.

Received: 6 January 2021 Revised: 12 May 2021 Accepted: 13 May 2021 Published online: 25 May 2021

\section{References}

1. Wang, C., Zhou, B. \& Xia, G. Mechanisms controlling germline cyst breakdown and primordial follicle formation. Cell Mol. Life Sci. 74, 2547-2566 (2017).

2. Pepling, M. E. From primordial germ cell to primordial follicle: mammalian female germ cell development. Genesis 44, 622-632 (2006).

3. Hsueh, A. J. Fertility: the role of mTOR signaling and KIT ligand. Curr. Biol. 24 R1040-R1042 (2014).

4. Qin, Y., Jiao, X., Simpson, J. L. \& Chen, Z. J. Genetics of primary ovarian insufficiency: new developments and opportunities. Hum. Reprod. Update 21, 787-808 (2015)

5. He, M. et al. LSD1 contributes to programmed oocyte death by regulating the transcription of autophagy adaptor SQSTM1/p62. Aging Cell 19, e13102 (2020)

6. Reddy, P., Zheng, W. \& Liu, K. Mechanisms maintaining the dormancy and survival of mammalian primordial follicles. Trends Endocrinol. Metab. 21, 96-103 (2010).

7. Sun, Y. C., Sun, X. F., Dyce, P. W., Shen, W. \& Chen, H. The role of germ cell loss during primordial follicle assembly: a review of current advances. Int. J. Biol. Sci. 13, 449-457 (2017).

8. Collins, G., Patel, B., Thakore, S. \& Liu, J. Primary ovarian Insufficiency: current concepts. South Med. J. 110, 147-153 (2017).

9. Tu, J. et al. Long non-coding RNAs in ovarian granulosa cells. J. Ovarian Res. 13, 63 (2020).

10. Stewart, J. Premature ovarian insufficiency is a lifelong condition. Clin. Endocrinol. 86, 168-169 (2017).

11. Tucker, E. J., Grover, S. R., Bachelot, A., Touraine, P. \& Sinclair, A. H. Premature ovarian insufficiency: new perspectives on genetic cause and phenotypic spectrum. Endocr. Rev. 37, 609-635 (2016).

12. Quinn, J. J. \& Chang, H. Y. Unique features of long non-coding RNA biogenesis and function. Nat. Rev. Genet. 17, 47-62 (2016).

13. Nachtergaele, S. \& He, C. The emerging biology of RNA post-transcriptional modifications. RNA Biol. 14, 156-163 (2017).

14. Wang, K. C. \& Chang, H. Y. Molecular mechanisms of long noncoding RNAs. Mol. Cell 43, 904-914 (2011).

15. Ernst, E. H., Nielsen, J., Ipsen, M. B., Villesen, P. \& Lykke-Hartmann, K. Transcriptome analysis of long non-coding RNAs and genes encoding paraspeckle proteins during human ovarian follicle development. Front. Cell Dev. Biol. 6, 78 (2018).

16. Li, J. et al. Increased new IncRNA-mRNA gene pair levels in human cumulus cells correlate with oocyte maturation and embryo development. Reprod. Sci. 22, 1008-1014 (2015).

17. Xiong, $Y$. et al. Cyclophosphamide promotes the proliferation inhibition of mouse ovarian granulosa cells and premature ovarian failure by activating the IncRNA-Meg3-p53-p66Shc pathway. Gene 596, 1-8 (2017).

18. Zheng, $\mathrm{L}$. et al. Differentially expressed IncRNAs after the activation of primordial follicles in mouse. Reprod. Sci. 26, 1094-1104 (2019).

19. Wang, X. et al. Long noncoding RNA HCP5 participates in premature ovarian insufficiency by transcriptionally regulating MSH5 and DNA damage repair via YB1. Nucleic Acids Res. 48, 4480-4491 (2020)

20. Zhao, J. et al. Polycystic ovary syndrome: novel and Hub IncRNAs in the insulin resistance-associated IncRNA-mRNA network. Front. Genet. 10, 772 (2019).

21. Yao, G. et al. Transcriptional profiling of long noncoding RNAs and their target transcripts in ovarian cortical tissues from women with normal menstrual cycles and primary ovarian insufficiency. Mol. Reprod. Dev. 86, 847-861 (2019).

22. Bartel, D. P. MicroRNAs: genomics, biogenesis, mechanism, and function. Cell 116, 281-297 (2004)

23. Kim, V. N. MicroRNA precursors in motion: exportin-5 mediates their nuclear export. Trends Cell Biol. 14, 156-159 (2004).

24. Ratti, M. et al. MicroRNAs (miRNAs) and long non-coding RNAs (IncRNAs) as new tools for cancer therapy: first steps from bench to bedside. Target Oncol. 15, 261-278 (2020).

25. Rupaimoole, R. \& Slack, F. J. MicroRNA therapeutics: towards a new era for the management of cancer and other diseases. Nat. Rev. Drug Discov. 16, 203-222 (2017).
26. Guo, Y., Sun, J. \& Lai, D. Role of microRNAs in premature ovarian insufficiency. Reprod. Biol. Endocrinol. 15, 38 (2017).

27. Zhang, $\mathrm{H}$. et al. microRNA 376a regulates follicle assembly by targeting Pcna in fetal and neonatal mouse ovaries. Reproduction 148, 43-54 (2014).

28. Wang, S. et al. MiR-125b regulates primordial follicle assembly by targeting activin receptor type $2 \mathrm{a}$ in neonatal mouse ovary. Biol. Reprod. 94, 83 (2016).

29. Li, T., Liu, X., Gong, X., E, Q., Zhang, X. \& Zhang, X. microRNA 92b-3p regulates primordial follicle assembly by targeting TSC1 in neonatal mouse ovaries. Cell Cycle 18, 824-833 (2019).

30. Alton, M. \& Taketo, T. Switch from BAX-dependent to BAX-independent germ cell loss during the development of fetal mouse ovaries. J. Cell Sci. 120 417-424 (2007)

31. Gawriluk, T. R. et al. Autophagy is a cell survival program for female germ cells in the murine ovary. Reproduction 141, 759-765 (2011).

32. Eskelinen, E. L., Reggiori, F., Baba, M., Kovács, A. L. \& Seglen, P. O. Seeing is believing: the impact of electron microscopy on autophagy research. Autophagy 7, 935-956 (2011).

33. Yu, Y., Nangia-Makker, P., Farhana, L. \& Majumdar, A. P. N. A novel mechanism of IncRNA and miRNA interaction: CCAT2 regulates miR-145 expression by suppressing its maturation process in colon cancer cells. Mol. Cancer 16, 155 (2017).

34. Yang, $X$. et al. PTBP3 splicing factor promotes hepatocellular carcinoma by destroying the splicing balance of NEAT1 and pre-miR-612. Oncogene 37, 6399-6413 (2018).

35. Dang, Y. et al. MicroRNA-22-3p is down-regulated in the plasma of Han Chinese patients with premature ovarian failure. Fertil. Steril. 103, 802-807 (2015).

36. Kuang, $\mathrm{H}$. et al. Profiling of differentially expressed microRNAs in premature ovarian failure in an animal model. Gynecol. Endocrinol. 30, 57-61 (2014).

37. Zhou, W., Xu, J., Wang, C., Shi, D. \& Yan, Q. miR-23b-3p regulates apoptosis and autophagy via suppressing SIRT1 in lens epithelial cells. J. Cell Biochem. 120, 19635-19646 (2019).

38. YiRen, $\mathrm{H}$. et al. Long noncoding RNA MALAT1 regulates autophagy associated chemoresistance via miR-23b-3p sequestration in gastric cancer. Mol. Cancer 16, 174 (2017).

39. Shi, J. Y., Chen, C., Xu, X. \& Lu, Q. miR-29a promotes pathological cardiac hypertrophy by targeting the PTEN/AKT/mTOR signalling pathway and suppressing autophagy. Acta Physiol. 227, e13323 (2019).

40. Vats, S. \& Manjithaya, R. A reversible autophagy inhibitor blocks autophagosome-lysosome fusion by preventing Stx17 loading onto autophagosomes. Mol. Biol. Cell 30, 2283-2295 (2019).

41. Itakura, E., Kishi-Itakura, C. \& Mizushima, N. The hairpin-type tail-anchored SNARE syntaxin 17 targets to autophagosomes for fusion with endosomes/ lysosomes. Cell 151, 1256-1269 (2012).

42. Tsuboyama, K. et al. The ATG conjugation systems are important for degradation of the inner autophagosomal membrane. Science 354, 1036-1041 (2016).

43. Kumar, S. et al. Mechanism of Stx17 recruitment to autophagosomes via IRGM and mammalian Atg8 proteins. J. Cell Biol. 217, 997-1013 (2018).

44. Yim, W. W. \& Mizushima, N. Lysosome biology in autophagy. Cell Discov. 6, 6 (2020).

45. Chapman, A. G., Cotton, A. M., Kelsey, A. D. \& Brown, C. J. Differentially methylated CPG island within human XIST mediates alternative P2 transcription and YY1 binding. BMC Genet. 15, 89 (2014).

46. Makhlouf, M. et al. A prominent and conserved role for $\mathrm{Y} Y 1$ in Xist transcriptional activation. Nat. Commun. 5, 4878 (2014).

47. Feng, L. et al. YY1-MIR372-SQSTM1 regulatory axis in autophagy. Autophagy 10. 1442-1453 (2014)

48. Yang, D. et al. YY1-PVT1 affects trophoblast invasion and adhesion by regulating mTOR pathway-mediated autophagy. J. Cell Physiol. 235, 6637-6646 (2020).

49. Zhao, X., Su, L., He, X., Zhao, B. \& Miao, J. Long noncoding RNA CA7-4 promotes autophagy and apoptosis via sponging MIR877-3P and MIR5680 in high glucose-induced vascular endothelial cells. Autophagy 16, $70-85$ (2020).

50. Wang, $K$. et al. APF IncRNA regulates autophagy and myocardial infarction by targeting miR-188-3p. Nat. Commun. 6, 6779 (2015).

51. Hendrich, B. D., Plenge, R. M. \& Willard, H. F. Identification and characterization of the human XIST gene promoter: implications for models of X chromosome inactivation. Nucleic Acids Res. 25, 2661-2671 (1997). 
52. Yoon, S. H. \& Choi, Y. M. Analysis of C43G mutation in the promoter region of the XIST gene in patients with idiopathic primary ovarian insufficiency. Clin. Exp. Reprod. Med. 42, 58-61 (2015).

53. Tang, R. et al. Mouse miRNA-709 directly regulates miRNA-15a/16-1 biogenesis at the posttranscriptional level in the nucleus: evidence for a microRNA hierarchy system. Cell Res. 22, 504-515 (2012)

54. Yi, R., Qin, Y., Macara, I. G. \& Cullen, B. R. Exportin-5 mediates the nuclear export of pre-microRNAs and short hairpin RNAs. Genes Dev. 17, 3011-3016 (2003).

55. Martinez, l. et al. An Exportin-1-dependent microRNA biogenesis pathway during human cell quiescence. Proc. Natl Acad. Sci. USA 114, E4961-E4970 (2017).
56. Zhang, Q., Chen, B., Liu, P. \& Yang, J. XIST promotes gastric cancer (GC) progression through TGF- $\beta 1$ via targeting miR-185. J. Cell Biochem. 119 2787-2796 (2018)

57. Chen, D. L. et al. Long non-coding RNA XIST regulates gastric cancer progression by acting as a molecular sponge of miR-101 to modulate EZH2 expression. J. Exp. Clin. Cancer Res. 35, 142 (2016).

58. Cheng, Q. \& Wang, L. LncRNA XIST serves as a ceRNA to regulate the expression of ASF1A, BRWD1M, and PFKFB2 in kidney transplant acute kidney injury via sponging hsa-miR-212-3p and hsa-miR-122-5p. Cell Cycle 19, 290-299 (2020) 